\title{
Malignant peritoneal mesothelioma diagnosed 50 years post-radiotherapy for ovarian cancer in a patient with a history of multiple malignancies: An autopsy case
}

\author{
MAKI OHYA $^{1,2}$, MIKIKO KOBAYASHI ${ }^{1}$, TOSHIRO SOZUKI $^{3}$, HIROYUKI KANNO $^{1}$ and KOH NAKAZAWA ${ }^{2}$ \\ ${ }^{1}$ Department of Pathology, Shinshu University School of Medicine, Matsumoto, Nagano 390-8621; \\ Departments of ${ }^{2}$ Laboratory Medicine and ${ }^{3}$ Respiratory Medicine, National Hospital Organization Matsumoto Medical Center, \\ Matsumoto, Nagano 399-8701, Japan
}

Received January 31, 2019; Accepted June 19, 2019

DOI: $10.3892 / \mathrm{mco} .2019 .1906$

\begin{abstract}
As the number of long-term cancer survivors is increasing, the incidence of post-irradiation malignant mesothelioma may also increase. We herein present the case of an 85-year-old female patient with a history of several surgeries for solid tumors and radiotherapy to the pelvis, who presented with abdominal pain and diarrhea. The patient's general condition gradually worsened and she succumbed to cardiopulmonary arrest triggered by vomiting $\sim 3$ months after the onset of the abdominal symptoms. An autopsy revealed malignant intestinal obstruction caused by peritoneal mesothelioma. Irradiation is a known risk factor for malignant mesothelioma, which may develop $\sim 10-30$ years after radiotherapy. To the best of our knowledge, this is the first report of a patient with malignant mesothelioma developing 50 years after radiotherapy. The aim of the present study was to remind physicians that malignant mesothelioma should be considered in the differential diagnosis of patients with a history of radiotherapy who present with gastrointestinal symptoms.
\end{abstract}

\section{Introduction}

Malignant mesothelioma is an aggressive neoplasm that arises from the mesothelial cells lining serosal surfaces. The majority of mesotheliomas arise in the pleura (85.5\%), and malignant peritoneal mesothelioma (MPM) is a rare tumor accounting for $13.2 \%$ of all malignant mesotheliomas (1). The incidence of MPM varies across countries, but there are reports that it ranges from 0.5 to 3 cases per 1 million population (2). According to the World Health Organization, the

Correspondence to: Dr Maki Ohya, Department of Pathology, Shinshu University School of Medicine, 3-1-1 Asahi, Matsumoto, Nagano 390-8621, Japan

E-mail:mendo@shinshu-u.ac.jp

Key words: malignant peritoneal mesothelioma, postoperative, radiotherapy age-specific mortality rate of peritoneal malignant mesothelioma is 0.3 per 1 million people, and the mean age at death of MPM is 66.0 years (3). However, as the number of patients with MPM is on the increase (2-4), clinicians and pathologists may acquire more experience with MPM patients in the future.

Asbestos exposure is the most important risk factor for malignant mesothelioma, including MPM (4). In addition, irradiation is also a known risk factor for malignant mesothelioma, and many develop $\sim 10$ to 30 years after radiotherapy (5-12). Other risk factors for malignant mesothelioma have been reported to include other minerals, such as erionite, thorium and mica $(2,4)$. However, as these other mineral-related risks are only reported in case reports, the relative risk of developing MPM has not yet been quantified (4).

We herein report a case of MPM diagnosed on autopsy in a patient who succumbed to intestinal obstruction. The patient had no history of asbestos exposure, but had a history of radiotherapy for ovarian cancer $\sim 50$ years earlier. To the best of our knowledge, this is the first report of a patient with malignant mesothelioma that developed this long after radiotherapy.

\section{Case report}

An 85-year-old Japanese woman presented at the Matsumoto Medical Center with abdominal pain and diarrhea in June, 2017. The patient had no history of asbestos exposure; however, she had a history of several tumor surgeries: Bilateral adnexectomy and postoperative radiotherapy for ovarian tumors in her 30s (details unknown), right upper lobectomy for lung adenocarcinoma at the age of 69 years, thymoma resection at the age of 73 years, and rectal amputation with artificial anostomy for rectal adenocarcinoma at the age of 79 years. The patient had not received postoperative treatment, including radiation therapy, following surgery for rectal cancer. A physical examination revealed bilateral pleural effusion, ascites, and lower leg edema. Laboratory tests revealed an increased white blood cell count (12,270/ $\mu \mathrm{l}$ : Reference value 3,300-8,600/ $\mu \mathrm{l})$, anemia (hemoglobin, $7.4 \mathrm{~g} / \mathrm{dl}$ : Reference value 11.6-14.8 g/dl), hypoalbuminemia (albumin, $1.4 \mathrm{~g} / \mathrm{dl}$ : Reference value 4.1-5.1 g/dl), and elevated C-reactive protein level $(13.3 \mathrm{mg} / \mathrm{dl}$ : Reference value $0.00-0.14 \mathrm{mg} / \mathrm{dl})$. The serum levels of tumor markers, 

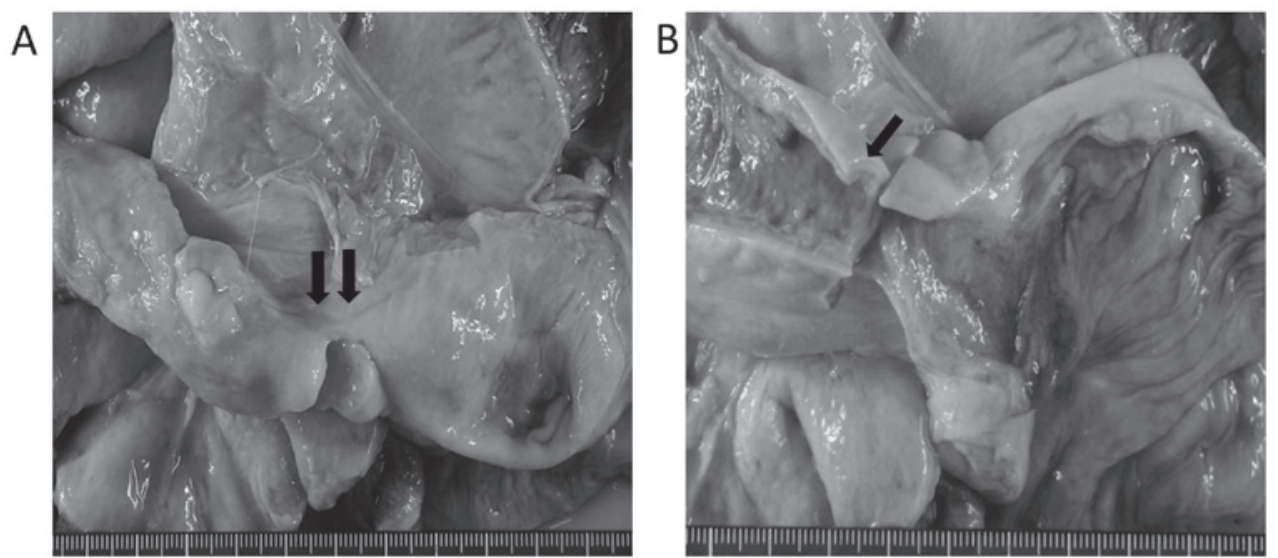

Figure 1. Macroscopic findings of the ileum. (A) Focal narrowing was observed over a length of $\sim 5 \mathrm{~cm}$ in the ileum. (B) The cut surface of the ileal narrowing revealed thickening of the intestinal wall (arrows).
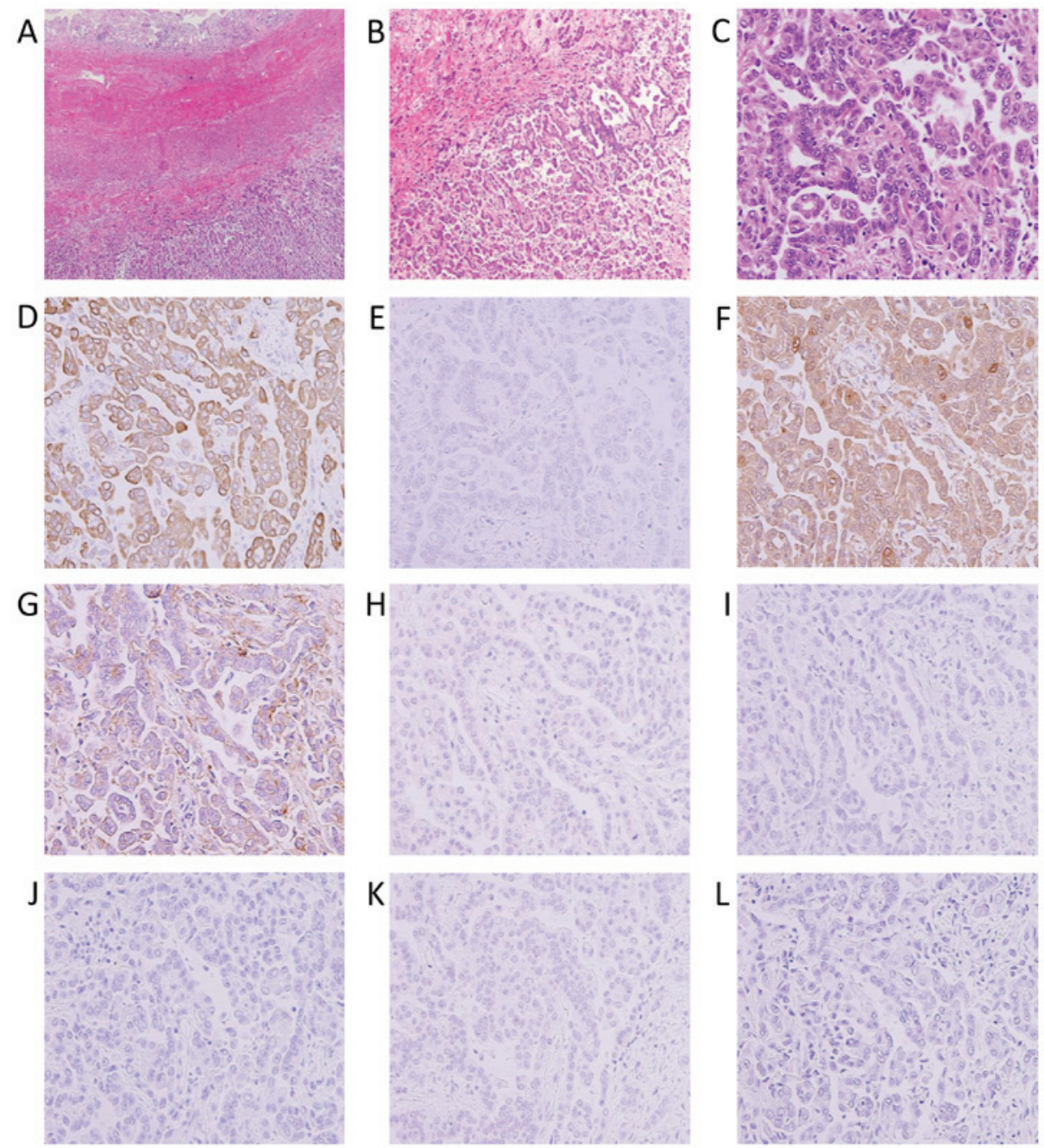

Figure 2. Histological findings of MPM. (A-C) Hematoxylin and eosin staining and (D-L) immunohistochemistry for (D) CK7, (E) CK20, (F) calretinin, (G) D2-40, (H) TTF-1, (I) CEA, (J) CA125, (K) ER and (L) PAX8. (A) The tumor invaded the subserosal tissue of the ileum and (B and C) was composed of epithelial cells arranged in a papilotubular pattern. These cells were positive for CK7, calretinin and D2-40, and negative for CK20, TTF-1, CEA, CA125, ER and PAX8. Magnification (A) x40, (B) x200 and (C-L) x400. MPM, malignant peritoneal mesothelioma; CK, cytokeratin; TTF, throid transcriprion factor; CEA, carcinoembryonic antigen; CA125, carbohydrate antigen 125; ER, estrogen receptor; PAX8, paired box 8.

including carcinoembryonic antigen (CEA) and carbohydrate antigen 125 (CA125), were not elevated. Therefore a postoperative adhesive ileus was suspected and the patient was treated with antibiotics and albumin supplementation, in addition to intravenous fluid administration, as neither the patient nor her family wished to have aggressive examinations or treatment. In 
addition to the presenting symptoms, vomiting, hematemesis and bleeding appeared in September, 2017. The patient's general condition gradually worsened, and she succumbed to cardiopulmonary arrest triggered by vomiting at $\sim 3$ months after the onset of the abdominal symptoms.

An autopsy was performed with the consent of the patient's family. Macroscopically, surgical scars were identified in the right chest and the lower abdominal midline. In the pleural cavity, clear yellowish pleural effusion $(350 \mathrm{ml}$ on the left side and $250 \mathrm{ml}$ on the right side) and pleural adhesions on the upper right side were observed. There was no evident pleural plaque formation. The abdominal cavity contained 3,000 ml of slightly cloudy, yellowish ascitic fluid, and moderate intestine-to-intestine and intestine-to-pelvic peritoneum adhesions were observed. The ileum exhibited adhesions to the pelvic wall with focal narrowing for a length of $\sim 5 \mathrm{~cm}$ (Fig. 1A). On cross section, the intestinal wall in the area of the ileal narrowing appeared thickened (Fig. 1B). On histopathological examination of the thickened ileal wall, proliferating atypical cells on the peritoneal surface were identified (Fig. 2A and B). These atypical cells had enlarged nuclei and were arranged in small papillary or tubular formations (Fig. 2C). No mucin production was observed. Immunohistochemically, the atypical cells were positive for cytokeratin (CK) 7 (Fig. 2D), calretinin (Fig. 2F) and podoplanin/D2-40 (Fig. 2G), but negative for CK20 (Fig. 2E), thyroid transcription factor-1 (Fig. 2H), CEA (Fig. 2I), CA125 (Fig. 2J), estrogen receptor (ER) (Fig. 2K), and paired box 8 (PAX8) (Fig. 2L). Therefore, the patient was diagnosed with MPM of the epithelioid type. MPM with fibrous thickening of the intestinal wall was limited to the ileum, but focally involved the serosa of the stomach, jejunum, and the fibrous capsule of the spleen. No recurrence or metastasis of the ovarian tumor, thymoma, lung adenocarcinoma, or colorectal adenocarcinoma were identified locally or systemically.

\section{Discussion}

Clinically, postoperative adhesive ileus and recurrence or metastasis of the prior cancers was suspected due to the patient's medical history. In general, the abdominal symptoms of MPM are non-specific, and may include ascites, retention, abdominal distension, abdominal pain, weight loss, nausea and vomiting (4). The imaging and macroscopic findings of MPM are also non-specific, such as peritoneal thickening and mass formation (4). Thus, it is difficult to clinically distinguish MPM from cancer recurrence or metastasis. ${ }^{18} \mathrm{~F}$-fluorodeoxyglucose-positron emission tomography (PET) was recently reported to be a valuable imaging tool in the preoperative diagnosis and management of MPM (13). In the future, detailed whole-body search, including PET examination, may be useful for the early diagnosis of MPM.

Histologically, MPM is classified into three subtypes: Epithelioid, sarcomatoid and biphasic. The majority of MPMs are of the epithelioid type, and display a papillotubular or solid pattern $(4,14)$. Thus, morphological differentiation between epithelioid-type MPM and adenocarcinoma is usually difficult. In particular, morphological differentiation between epithelioid MPM and serous adenocarcinoma, which arises in the uterus, ovary, oviduct and the peritoneum, is crucial in female patients (15). Immunohistochemical examination is necessary for definitive diagnosis. MPM is generally positive for mesothelial markers (calretinin, D2-40, Wilms' tumor-1 and $\mathrm{CK}$ 5/6) and negative for epithelial markers (several cytokeratins, such as CK AE1/AE3, CK7 and CAM 5.2), adenocarcinoma markers (CEA, CA 19-9 and epithelial-specific antigen/Ber-EP 4), and hormone receptors (ER and progesterone receptor) $(4,14,15)$. In addition, the Müllerian marker PAX8 is highly positive in serous adenocarcinoma, but generally negative in MPM $(14,16)$. Immunohistochemical analysis using a panel of multiple markers is recommended for accurate diagnosis. In the present case, surgery for the ovarian tumor had been performed $>50$ years earlier and details, including the histological subtype, were unavailable; however, immunohistochemical examination (positive for calretinin and D2-40, and negative for PAX8, CA125 and ER) confirmed the diagnosis of MPM and excluded serous adenocarcinoma.

Asbestos exposure is the most common cause of malignant mesothelioma, including MPM, particularly in men $(1,4,14)$. However, there are several cases in women that are not associated with asbestos exposure (4). Our patient had a negative history for occupational asbestos exposure, as she was a housewife. Furthermore, none of her family members have developed asbestos-related diseases, such as mesothelioma and lung cancer, to date. In addition, findings indicating asbestos exposure, such as pleural plaque formation, were not observed on autopsy. Therefore, exposure to asbestos was an unlikely cause of MPM in this case.

However, the patient had a history of multiple maligancies (ovarian cancer, lung cancer, thymoma and rectal cancer) and radiotherapy to the pelvis. In this case, although the patient's history suggested the possibility of a genetic mutation that increases susceptibility to developing multiple malignancies, the main lesion of MPM was located in the ileum and adhered to the pelvis, with superficial spread to a limited serosal area of the stomach, jejunum and spleen; the location of the lesion corresponded to the pelvic irradiation site following ovarian cancer surgery. Malignant mesothelioma can occur after radiotherapy for tumors, which suggests that direct irradiation may be a risk factor for its development. Malignant mesotheliomas following radiation therapy usually develop $\sim 10-30$ years after radiotherapy (5-12). In addition, the US epidemiological study on radiotherapy (external radiation) for solid tumors suggested that direct irradiation was associated with the onset of malignant mesothelioma (17). In particular, there is an increased risk for $>10$ years after irradiation (17). However, the cumulative incidence of malignant mesothelioma was lower over a time period of $>40$ years after irradiation (17). This may be due to the fact that malignant mesothelioma is a rare disease, and other clinical factors, such as the onset of other diseases, are involved in long-term epidemiological surveys. As described above, there have been several case reports of MPM developing after radiation therapy. However, there are differences in the clinical data described in each of those cases. Furthermore, in the present case, the tissue type of the primary lesion, the irradiation dose and the duration of the radiation therapy were unknown, as this was an event from 50 years ago and the medical records had been discarded. To the best of our knowledge, there have been no reports to date of MPM developing 50 years after radiotherapy. However, 
the population in Japan is aging, and the number of patients with malignant mesothelioma is increasing annually (18). Although a number of malignant mesotheliomas were associated with asbestos exposure in the 1970s, some may have been related to radiotherapy. As the long-term prognosis after solid tumor surgery with radiotherapy is expected to increase due to the recent advances in medical technology, the incidence of post-irradiation malignant mesothelioma may also increase. In addition, long-term cancer survivors often develop other cancers, which makes diagnosis more difficult, as in the current case; however, malignant mesothelioma should be considered in the differential diagnosis if a patient has a history of radiotherapy.

\section{Acknowledgements}

Not applicable.

\section{Funding}

No funding was received.

\section{Availability of data and materials}

The datasets during and/or analyzed during the present study available from the corresponding author on reasonable request.

\section{Authors' contributions}

MO and MK designed the study. MO wrote the manuscript and assessed the figures and tables. MK, TS, HK and KN critically revised the manuscript and were involved in data interpretation. MO and MK finalized the manuscript and submitted the paper for publication. All authors have edited the manuscript for intellectual content. All authors have read and approved the final version of this manuscript for publication.

\section{Ethics approval and consent to participate}

Not applicable.

\section{Patient consent for publication}

The patient and her family provided written informed consent for the publication of the case details and any associated images.

\section{Competing interests}

The authors declare that they have no competing interests.

\section{References}

1. Gemba K, Fujimoto N, Aoe K, Kato K, Takeshima Y, Inai K and Kishimoto T: Treatment and survival analyses of malignant mesothelioma in Japan. Acta Oncol 52: 803-808, 2013.

2. Boffetta P: Epidemiology of peritoneal mesothelioma: A review. Ann Oncol 18: 985-990, 2007.

3. Delgermaa V, Takahashi K, Park EK, Le GV, Hara T and Sorahan T: Global mesothelioma deaths reported to the World Health Organization between 1994 and 2008. Bull World Health Organ 89: 716-724, 724A-724C, 2011.

4. Kim J, Bhagwandin S and Labow DM: Malignant peritoneal mesothelioma: A review. Ann Transl Med 5: 236, 2017.

5. Babcock TL, Powell DH and Bothwell RS: Radiation-induced peritoneal mesothelioma. J Surg Oncol 8: 369-372, 1976.

6. Antman KH, Corson JM, Li FP, Greenberger J, Sytkowski A, Henson DE and Weinstein L: Malignant mesothelioma following radiation exposure. J Clin Oncol 1: 695-700, 1983.

7. Hofmann J, Mintzer D and Warhol MJ: Malignant mesothelioma following radiotherapy. Am J Med 97: 379-382, 1994.

8. Kinutani M, Nagai N, Kurihara K, Sakata K, Tanimoto H, Murakami T, Takehara K, Takenaka M, Okamoto E and Ohama K: A case of malignant mesothelioma arising from uterine serosa after radiation therapy in uterine cervical cancer. Nihon Sanka Fujinka Gakkai Zasshi 46: 911-914, 1994 (In Japanese).

9. Cavazza A, Travis LB, Travis WD, Wolfe JT III, Foo ML, Gillespie DJ, Weidner $\mathrm{N}$ and Colby TV: Post-irradiation malignant mesothelioma. Cancer 77: 1379-1385, 1996.

10. Sato F, Yamazaki H, Ataka K, Mashima I, Suzuki K, Takahashi T, Umezu H and Gejyo F: Malignant peritoneal mesothelioma associated with deep vein thrombosis following radiotherapy for seminoma of the testis. Intern Med 39: 920-924, 2000.

11. Beier KM, Gallup DG, Burgess R and Stock RJ: Occurrence of malignant peritoneal mesothelioma after surgery and irradiation for cervical cancer. Gynecol Oncol 17: 375-380, 1984.

12. Horie A, Hiraoka K, Yamamoto O, Haratake J, Tsuchiya T and Sugimoto H: An autopsy case of peritoneal malignant mesothelioma in a radiation technologist. Acta Pathol Jpn 40: 57-62, 1990.

13. Domènech-Vilardell A, Rasiej MJ, Taub RN and Ichise M: Clinical utility of 18F-FDG positron emission tomography in malignant peritoneal mesothelioma. Q J Nucl Med Mol Imaging 60: 54-61, 2016.

14. Husain AN, Colby TV, Ordonez NG, Allen TC, Attanoos RL, Beasley MB, Butnor KJ, Chirieac LR, Churg AM, Dacic S, et al: Guidelines for pathologic diagnosis of malignant mesothelioma. 2017 Update of the consensus statements from the International Mesothelioma Interest Group: Arch Pathol Lab Med 142: 89-108, 2018.

15. Baker PM, Clement PB and Young RH: Malignant peritoneal mesothelioma in women: A study of 75 cases with emphasis on their morphologic spectrum and differential diagnosis. Am J Clin Pahol 123: 724-737, 2005.

16. Takeshima Y, Inai K, Amatya VJ, Gemba K, Aoe K, Fujimoto N, Kato $\mathrm{K}$ and Kishimoto T: Accuracy of pathological diagnosis of mesothelioma cases in Japan: Clinicopathological analysis of 382 cases. Lung Cancer 66: 191-197, 2009.

17. Farioli A, Ottone M, Morganti AG, Compagnone G, Romani F, Cammelli S, Mattioli S and Violante FS: Radiation-induced mesothelioma among long-term solid cancer survivors: A longitudinal analysis of SEER database. Cancer Med 5: 950-959, 2016.

18. Gemba K, Fujimoto N, Kato K, Aoe K, Takeshima Y, Inai K and Kishimoto T: National survey of malignant mesothelioma and asbestos exposure in Japan: Cancer Sci 103: 483-490, 2012. 